\title{
PROGRAMA DE GERENCIAMENTO DE RESÍDUOS DOS LABORATÓRIOS DE GRADUAÇÃO DA UNIVERSIDADE REGIONAL INTEGRADA DO ALTO URUGUAI E DAS MISSÕES - CAMPUS ERECHIM
}

\author{
Anelise Schwengber Demaman, Suzana Funk, Luiz Ubiratan Hepp, Alexandro Magno dos Santos Adário e Sibele Berenice \\ Castellã Pergher* \\ Centro Tecnológico, Universidade Regional Integrada do Alto Uruguai e das Missões, Campus Erechim, CP 743, \\ 99700-000 Erechim - RS
}

Recebido em 12/8/03; aceito em 15/12/03; publicado na web em 27/05/04

\begin{abstract}
A PROGRAM FOR MANAGING RESIDUES FROM TEACHING LABORATORIES OF THE INTEGRATED REGIONAL UNIVERSITY OF ALTO URUGUAI E DAS MISSÕES - CAMPUS ERECHIM. This work presents a proposal for the management of residues from teaching laboratories. The main goals of the proposal are: scale reduction of experiments, reuse of residues as raw materials for new experiments and appropriate treatment and storage of residues. The methodology includes standardized labels for residue classification and registration of experimental classes and their residues in files. The management seemed to be efficient, resulting in a reduction of the amount of reagents utilized and residues generated, and an increase of reutilization of residues. A considerable decrease of needed storage space and suitable methods for correct residue disposal were achieved. We expect that all laboratories, including those exclusively for research activities, become involved, in a near future, in the Residue Management Project of URI - Campus Erechim.
\end{abstract}

Keywords: management of residues; environmental chemistry; laboratory.

\section{INTRODUÇÃO}

Recentemente vem crescendo o interesse pela Química Limpa, através da qual as indústrias estão gradualmente trocando processos tradicionais por tecnologias ambientalmente corretas, sendo que as universidades estão montando programas de gerenciamentos de resíduos $^{1-7}$. Essas atitudes são essenciais para que os danos ambientais e os riscos à saúde sejam minimizados. A implementação destes programas possibilita que os resíduos recuperados possam não somente ser sucessivamente reutilizados no mesmo processo em que foram gerados, como também ser transformados em matéria prima. Em vista disso, este trabalho apresenta um programa de Gerenciamento de Resíduos.

Os objetivos são a recuperação e reutilização dos resíduos de laboratórios visando minimizar os rejeitos. As metas a serem alcançadas são redução de escala dos experimentos; reaproveitamento do resíduo como insumo; acondicionamento adequado dos rejeitos e tratamento dos rejeitos para o descarte. Com estas metas visa-se a diminuição do consumo de reagentes, a redução da produção de resíduos, o aumento do reaproveitamento dos resíduos, a diminuição dos rejeitos acondicionados e um descarte adequado dos rejeitos.

\section{HISTÓRICO}

Quando o Curso de Química da URI - Campus Erechim foi implantado, no segundo semestre de 2001, já existia no campus uma Estação de Tratamento de Efluentes - ETE (desde 1997) destinada ao tratamento de todos os resíduos líquidos produzidos nos laboratórios do Centro Tecnológico e do Centro de Ciências da Saúde. O sistema de funcionamento da ETE é baseado em reações de coagulações e floculação, utilizando sulfato de alumínio e polímero, sendo que o material sólido é separado por decantação e o lodo produzido

*e-mail: pergher@uri.com.br é recolhido em leitos de secagem para posterior destinação. De forma geral, o sistema possui um rendimento de aproximadamente $70 \%$ quando se trata de parâmetros indicadores de matéria orgânica e de $80 \%$ para metais pesados, o que possibilita a emissão de um resíduo dentro dos padrões legais estabelecidos por legislação para o corpo hídrico receptor. O procedimento adotado, ao término das aulas práticas, consistia no descarte dos rejeitos pela pia, sendo que os mesmos eram conduzidos, por tubulação própria, ao tanque de aeração e neutralização da ETE para início do processo de tratamento. Embora não haja registro de acidentes, é possível que metais pesados ou compostos mais tóxicos, que mesmo com o $\mathrm{pH}$ neutro, podem ter chegado ao rio, sem mencionar que resíduos a caminho da ETE podiam reagir com outras substâncias nas tubulações, podendo ocasionar acidentes. Muitos desses compostos descartados na pia podem ser reutilizados obtendo-se, assim, economia financeira. Considerando essa vantagem econômica e a menor agressão ao meio ambiente, foi realizado um curso (com a participação de professores do Curso de Química, alunos e funcionários) intitulado “Gerenciamento de Rejeitos Químicos Perigosos em Instituições de Ensino e Pesquisa", ministrado por Annelise E. Gerbase e Édison S. de Melo do IQUFRGS, em julho de 2002. Neste curso, os participantes desenvolveram vários trabalhos na área de gerenciamento e a partir destes, foi proposto um programa de gerenciamento de resíduos para a URI Campus Erechim.

\section{O SISTEMA ATUAL}

O Centro Tecnológico da URI - Campus Erechim conta com uma Central de Materiais, onde ficam armazenados os reagentes, resíduos, vidrarias e outros materiais comuns de laboratório. Antes de cada aula prática, o professor responsável preenchia um formulário (Figura 1) através do qual solicitava todo o material necessário para a execução da mesma. Com esse sistema existia um controle de reagentes gastos para sua futura compra e uma otimização do uso das vidrarias e reagentes, evitando a subutilização destes. 


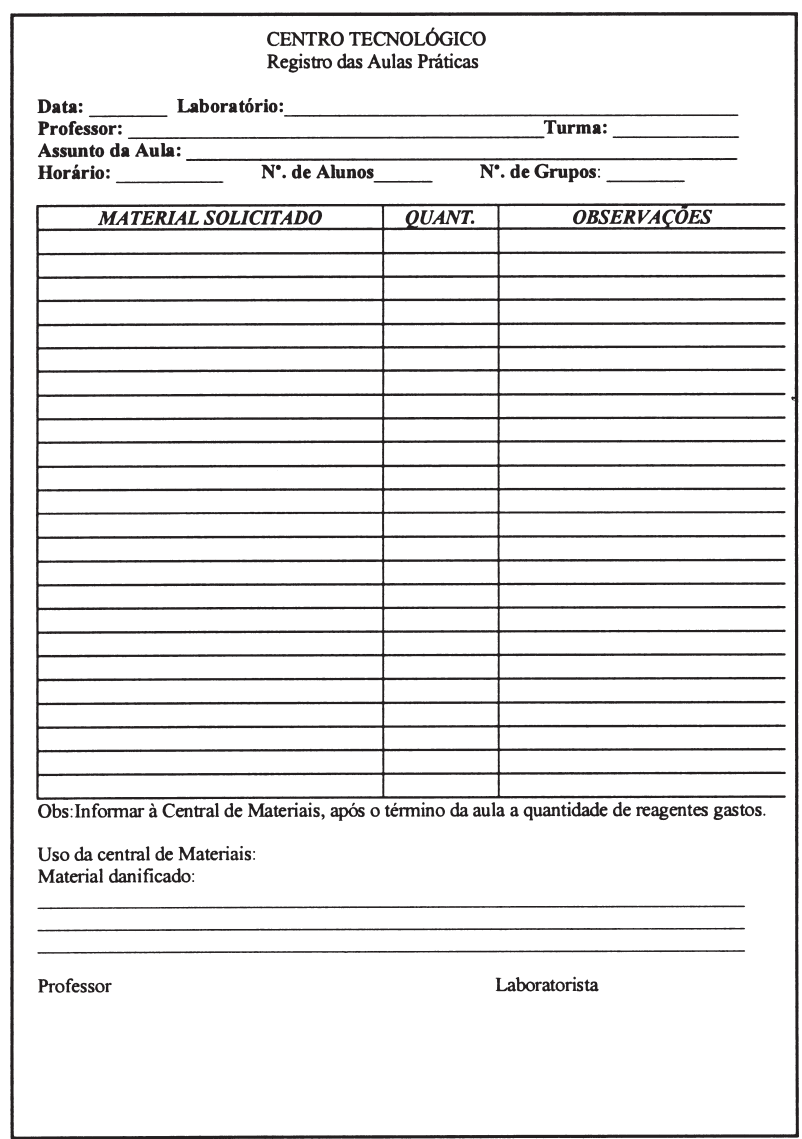

Figura 1. Formulário (antigo) de solicitação das aulas práticas

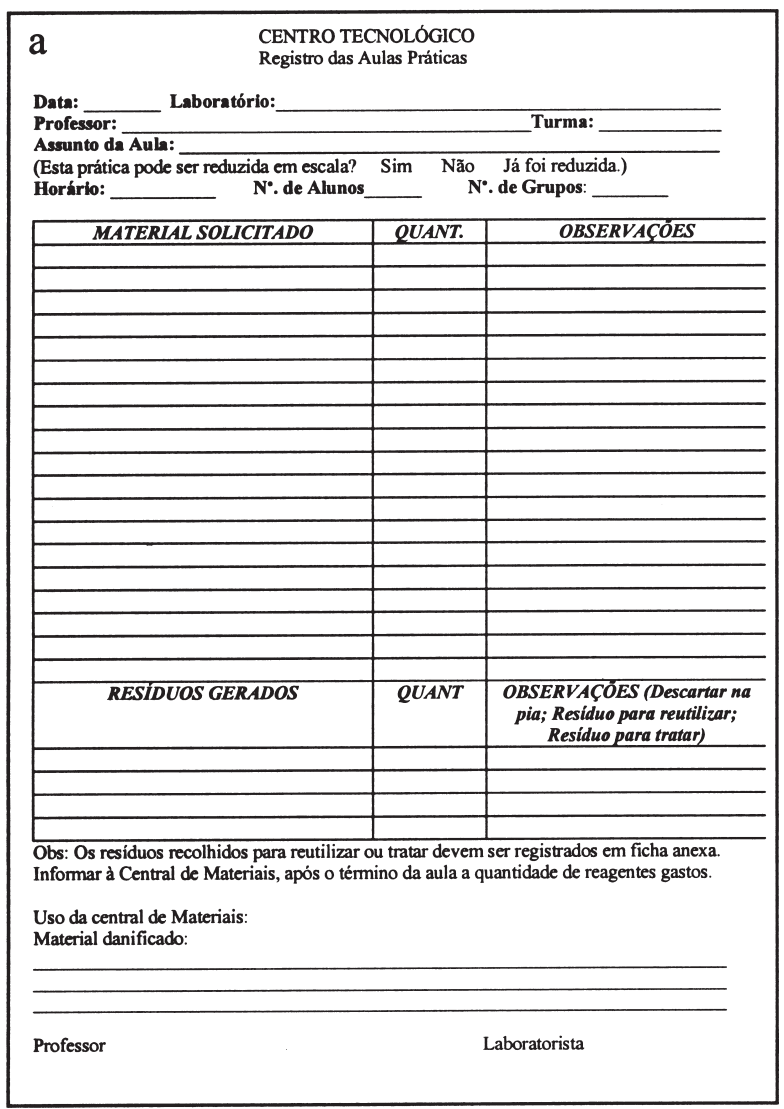

Na Central de Materiais, trabalham 4 funcionários e 3 estagiários que, entre outras atividades, são encarregados de montar as aulas práticas indicadas pelos professores. $\mathrm{O}$ controle existente era o de reagentes e materiais utilizados nestas atividades, não existindo um monitoramento qualitativo e quantitativo dos resíduos gerados, pois os mesmos eram descartados na pia. Outro problema existente era o acondicionamento inadequado dos produtos preparados ou rejeitos produzidos.

\section{O SISTEMA PROPOSTO}

Utilizando o formulário de registro das aulas práticas já existente no Centro Tecnológico, foi proposto um novo formulário (Figura 2a) no qual foram acrescentados alguns itens. Primeiramente foi incluída a pergunta: "Esta prática pode ser reduzida em escala?" pela qual o professor responsável pela prática é estimulado a pensar em uma possível redução de escala e, conseqüentemente, proporcionar a redução no consumo de reagentes e na produção de resíduos. Com um levantamento destas respostas podemos verificar se o programa está fomentando a adesão dos professores. A este formulário foram adicionadas, na tabela, a solicitação de quais resíduos são gerados na prática, sua quantidade e se podem ser descartados na pia, reutilizados ou se devem ser tratados para descarte. Acrescentamos ainda uma observação informando que os resíduos recolhidos, tanto para tratamento como para reutilização, devem ser registrados em um formulário anexo (Figura 2b). O formulário de Registro de Resíduos proposto pede informações mais detalhadas sobre o resíduo e o processo que o gerou, tais como reagentes utilizados, reações, composição do resíduo, classificação e características do resíduo quanto à periculosidade. Pedimos também uma proposta de reutilização e/ ou tratamento para tal resíduo. Com estes formulários estamos for-

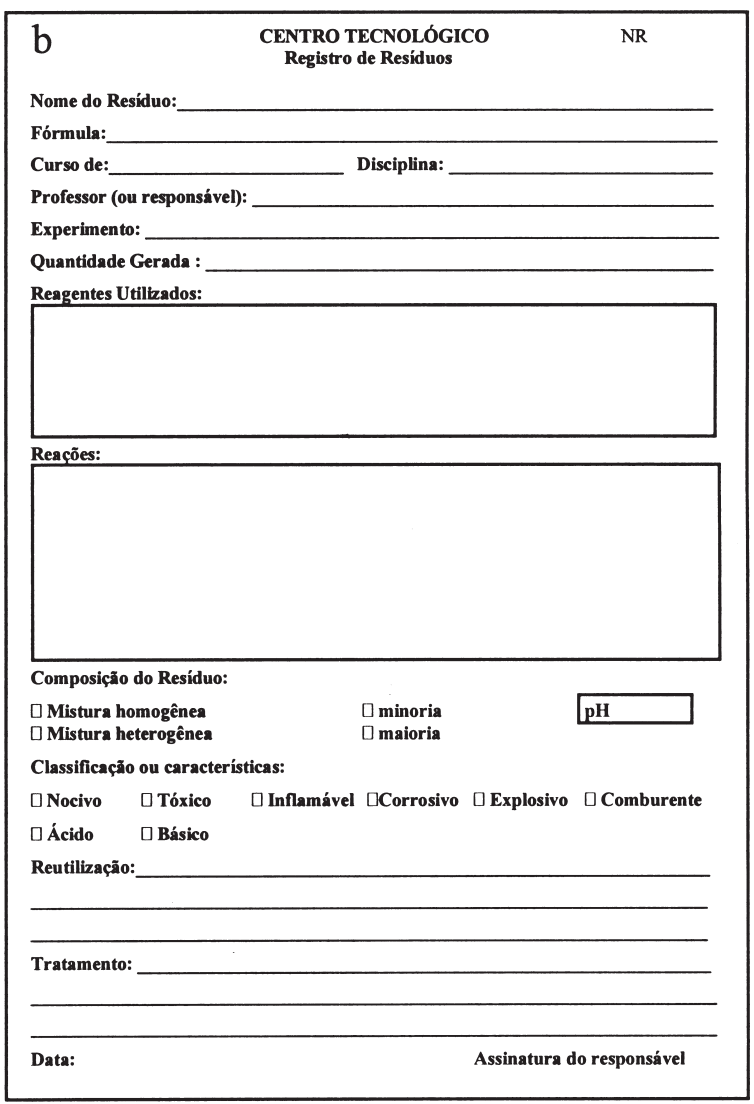

Figura 2. Formulários de registro das aulas práticas (a) e registro de resíduos (b) 
mando um cadastro de resíduos que servirá para controle e reaproveitamento do mesmo, o gerenciamento propriamente dito.

Como primeira proposta de gerenciamento resolvemos trabalhar com a disciplina Química Geral Experimental, a qual teve suas práticas analisadas uma a uma, levando em consideração os reagentes e as quantidades utilizadas, os resíduos gerados e quais destes resíduos poderiam ser reutilizados, na própria disciplina ou em outras. A Tabela 1 apresenta estes resultados.

É possível observar que a maioria dos resíduos gerados pode ser reutilizada como insumo na disciplina, outros podem ser utilizados em outras disciplinas e alguns não possuem uma utilização definida. Há dois rejeitos que devem ser tratados e descartados.
Como simbologia adotamos as cores Verde, Amarelo e Vermelho fazendo uma analogia com o semáforo de trânsito (sistema já adotado no IQ da $\mathrm{UFRGS}^{1}$ ). Cada cor possui o seguinte significado: $>$ Verde é um INSUMO, definido como produto originado de qualquer processo de recuperação ou de algum processo de síntese e que já possui destino de reutilização.

$>$ Amarelo é um RESÍDUO que pode ser reaproveitado em outro experimento.

> Vermelho é um REJEITO que é um resíduo, que não apresenta utilidade alguma, e precisa ser tratado e descartado.

Com base neste esquema de cores, foram propostos Rótulos (Figura 3) que apresentam na parte superior uma barra com a classifica-

Tabela 1. Proposta de gerenciamento por aulas práticas da disciplina Química Geral Experimental

\begin{tabular}{|c|c|c|c|}
\hline Experiência & Reagentes & Resíduos & Utilização \\
\hline Exp01 & Sem & Não gera & \\
\hline \multirow[t]{2}{*}{ Exp02 } & \multirow{2}{*}{$\mathrm{BaCl}_{2} ; \mathrm{Na}_{2} \mathrm{CO}_{3}$} & $\mathrm{NaCl}$ & Descartar na pia \\
\hline & & $\mathrm{BaCO}_{3}(\mathrm{~s})$ & Resíduo \\
\hline \multirow[t]{2}{*}{ Exp03 } & $\mathrm{NaOH}$ p.a.; $\mathrm{HCl}$ conc.; & $\mathrm{HCl} 1 \mathrm{~mol} / \mathrm{L}$; HCl 0,1 mol/L; & Insumos Exp 04, 10 e 14 \\
\hline & $\mathrm{NaOH} 0,1 \mathrm{~mol} / \mathrm{L}$ & $\mathrm{NaOH} 0,1 \mathrm{~mol} / \mathrm{L}$ & \\
\hline \multirow[t]{2}{*}{ Exp04 } & $\mathrm{HCl}$ 0,1 mol/L padroniz.; & $\mathrm{NaOH}$ bureta $0,1 \mathrm{~mol} / \mathrm{L}$ & Neutralizar e descartar na pia \\
\hline & $\mathrm{NaOH} 0,1 \mathrm{~mol} / \mathrm{L}$ & $\mathrm{HCl}$ bureta $0,1 \mathrm{~mol} / \mathrm{L}$ & \\
\hline Exp05 & Mistura $\mathrm{ZnO}_{2}+\mathrm{NaCl}$ & $\mathrm{ZnO}_{2(\mathrm{~s})} \quad \mathrm{NaCl}_{(\mathrm{s})}$ & Insumos Exp05 \\
\hline Exp06 & Clorofórmio & Clorofórmio & Insumo Exp06 \\
\hline \multirow[t]{3}{*}{ Exp07 } & Metais $\mathrm{Cu}, \mathrm{Mg}, \mathrm{Zn}$; & Sólidos & Rejeito \\
\hline & $\mathrm{CuSO}_{4} ; \mathrm{ZnCl}_{2} ; \mathrm{FeSO}_{4}$ & Soluções aquosas de cátions & Insumo para Analítica Qualita \\
\hline & $\mathrm{HgCl}_{2} ; \mathrm{AgNO}_{3}$ & & \\
\hline Exp08 & Fitas de $\mathrm{Mg} ; \mathrm{HCl} 6 \mathrm{~mol} / \mathrm{L}$ & $\mathrm{MgCl}_{2(\mathrm{aq})}$ & Descartar na pia \\
\hline \multirow[t]{3}{*}{ Exp09 } & $\mathrm{Na}_{2} \mathrm{~S}_{2} \mathrm{O}_{3} 0,1 \mathrm{~mol} / \mathrm{L}$ & $\mathrm{Na}_{2} \mathrm{SO}_{4}+\mathrm{S}$ & Química Inorgânica \\
\hline & $\mathrm{H}_{2} \mathrm{SO}_{4} 0,2 \mathrm{~mol} / \mathrm{L}$ & $\mathrm{Fe}(\mathrm{SCN})_{2 ;} \mathrm{NaSCN}$ & Resíduo; \\
\hline & $\mathrm{Fe}(\mathrm{SCN})_{3} ; \mathrm{CuSO}_{4}$ & $\mathrm{Na}_{2} \mathrm{~S}_{4} \mathrm{O}_{6} \mathrm{CuSO}_{4}$ & Rejeito \\
\hline \multirow[t]{4}{*}{ Exp10 } & $\mathrm{NH}_{4} \mathrm{CNS}_{(\mathrm{s}) ;} \mathrm{NH}_{4} \mathrm{Cl}_{(\mathrm{s})}$ & $\mathrm{NH}_{4} \mathrm{CNS}_{(\mathrm{aq}) ;} \mathrm{NH}_{4} \mathrm{Cl}_{(\mathrm{aq}) ;} \mathrm{FeCl}_{3(\mathrm{aq})}$ & Insumo Exp10 \\
\hline & $\mathrm{FeCl}_{3(\mathrm{~s}) ;} \mathrm{K}_{2} \mathrm{CrO}_{4}$ & $\left.\mathrm{NH}_{4} \mathrm{CNS} ; \mathrm{NH}_{4} \mathrm{Cl} ; \mathrm{FeCl}_{3} ; \mathrm{NH}_{4}\right)_{3} \mathrm{Fe}(\mathrm{CNS})_{6}$ & Rejeito \\
\hline & $\mathrm{Na}_{2} \mathrm{Cr}_{2} \mathrm{O}_{7} ; \mathrm{BaCl}_{2}$ & $\mathrm{CrO}_{4}^{2-} ; \mathrm{Cr}_{2} \mathrm{O}_{7}^{2-;} \mathrm{BaCrO}_{4(\mathrm{~s})}$ & Resíduos \\
\hline & $\mathrm{NaOH} ; \mathrm{HCl}$ & & \\
\hline \multirow[t]{2}{*}{ Exp11 } & $\mathrm{Co}\left(\mathrm{NO}_{3}\right)_{2} 0,5 \mathrm{~mol} / \mathrm{L} ; \mathrm{Co}\left(\mathrm{NO}_{3}\right)_{2(\mathrm{~s})}$ & $\mathrm{AgCl}_{(\mathrm{s})} \mathrm{Co}^{+2}, \mathrm{Cl}^{-}, \mathrm{CoCl}_{4}^{2-}$ & Resíduos \\
\hline & $\mathrm{AgNO}_{3} 0,2 \mathrm{~mol} / \mathrm{L} ; \mathrm{HCl}$ conc; $\mathrm{NaCl}_{(\mathrm{s})}$ & & \\
\hline \multirow[t]{2}{*}{ Exp12 } & \multirow{2}{*}{$\mathrm{CH}_{3} \mathrm{COOAg}_{(\mathrm{s})} ;$ Fio de $\mathrm{Cu}$} & $\mathrm{Ag}$ & $\mathrm{AgNO}_{3}$ Insumo Exp07e11; \\
\hline & & $\mathrm{Cu}^{+2}, \mathrm{CH}_{3} \mathrm{COO}^{-}$ & Resíduo \\
\hline \multirow[t]{4}{*}{ Exp13 } & $\mathrm{NaCl}_{(\mathrm{s})} ; \mathrm{CH}_{3} \mathrm{COONa}_{(\mathrm{s})}$ & $\mathrm{NaCl}_{\text {(aq) }} ; \mathrm{CH}_{3} \mathrm{COONa}_{\text {(aq) }} ;$ & Insumo Exp 07,13, 14 \\
\hline & $\mathrm{CuSO}_{4} \cdot 5 \mathrm{H}_{2} \mathrm{O}_{(\mathrm{s})} ; \mathrm{Na}_{2} \mathrm{~S} 1 \mathrm{~mol} / \mathrm{L}$ & $\mathrm{CuSO}_{4(\mathrm{aq}) ;} \mathrm{Na}_{2} \mathrm{~S} 1 \mathrm{~mol} / \mathrm{L}$; & \\
\hline & $\mathrm{KAl}\left(\mathrm{SO}_{4}\right)_{2} 0,01 \mathrm{~mol} / \mathrm{L}$ & $\mathrm{KAl}\left(\mathrm{SO}_{4}\right)_{2} 0,01 \mathrm{~mol} / \mathrm{L}$ & \\
\hline & $\mathrm{Na}_{2} \mathrm{CO}_{3} 0,1 \mathrm{~mol} / \mathrm{L}$ & $\mathrm{Na}_{2} \mathrm{CO}_{3} 0,1 \mathrm{~mol} / \mathrm{L}$ & \\
\hline \multirow[t]{5}{*}{ Exp14 } & $\mathrm{CH}_{3} \mathrm{COOH} 1$ e $0,2 \mathrm{~mol} / \mathrm{L}$ & Ácidos e Bases & Neutralizar e descartar na pia \\
\hline & $\mathrm{CH}_{3} \mathrm{COONa} 0,2 \mathrm{~mol} / \mathrm{L}$ & & \\
\hline & $\mathrm{NH}_{3} 0,5$ e $0,2 \mathrm{~mol} / \mathrm{L}$ & & \\
\hline & $\mathrm{NH}_{4} \mathrm{Cl}$ 0,2mol$/ \mathrm{L} ; \mathrm{HCl} 0,1 \mathrm{~mol} / \mathrm{L} ;$ & & \\
\hline & $\mathrm{NaOH} 0,1 \mathrm{~mol} / \mathrm{L}$ & & \\
\hline \multirow[t]{2}{*}{ Exp15 } & \multirow[t]{2}{*}{$\mathrm{HCl} 1 \mathrm{~mol} / \mathrm{L} ; \mathrm{NaOH} 1 \mathrm{~mol} / \mathrm{L} ; \mathrm{NaOH}_{(\mathrm{s})}$} & $\mathrm{NaCl}_{(\mathrm{aq})}$ & Descartar na pia; \\
\hline & & $\mathrm{NaOH} 1 \mathrm{~mol} / \mathrm{L}$ & Insumo Exp14 \\
\hline
\end{tabular}



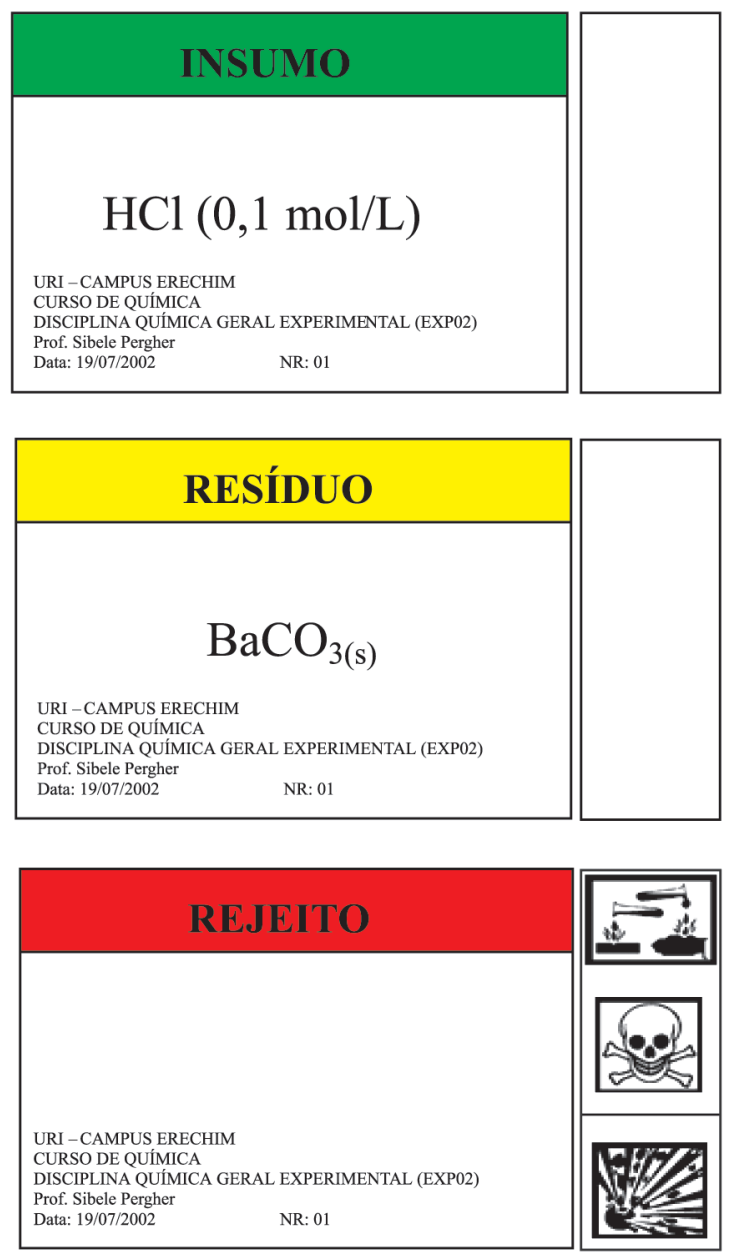

Figura 3. Proposta de rótulos dos resíduos

ção (Insumo, Resíduo e Rejeito), e na parte lateral do rótulo, um espaço para a indicação dos símbolos de toxidade. Assim, é rapidamente identificado o tipo de resíduo e sua toxidade. O rótulo possui também outras informações como disciplina e curso no qual foi gerado o resíduo, o professor responsável, o número de registro de resíduo e a data.

\section{RESULTADOS}

Esta proposta foi implementada em julho de 2002, juntamente com a padronização dos rótulos de identificação. A disciplina de Química Geral Experimental utiliza o gerenciamento proposto na Tabela 1. Outras disciplinas do Curso de Química também propuseram seu gerenciamento de resíduos e o utilizam, tais como Química Analítica Clássica e Química Inorgânica 2.

Os resíduos não foram mais descartados sem critério e iniciouse sua estocagem. A reutilização dos resíduos está em operação, e houve uma diminuição expressiva dos resíduos estocados que tinham reutilização. Isso contribuiu para melhoria no rendimento da ETE, que passou a ter menor diversidade de substâncias, graças aos funcionários da Central de Materiais que, buscando eliminar os resíduos estocados, fazem o cruzamento entre as necessidades dos professores e os resíduos estocados, providenciando a substituição dos reagentes solicitados. Eles conseguem identificar os resíduos para reutilização, visualizando aqueles que possuem os rótulos verdes ou amarelos.

Os resíduos destinados ao tratamento para descarte (rótulos vermelhos) precisam ser catalogados para poderem ser trabalhados e descartados. Outro problema deste processo é a falta de preenchimento dos formulários, sendo que alguns professores não os preenchem corretamente ou fazem a solicitação de materiais para aulas práticas através de pedidos em papel comum. Mesmo aqueles que eram preenchidos corretamente, muitas vezes não continham informações detalhadas sobre quais resíduos foram gerados ou como tratar esses resíduos.

A falta de um controle informatizado dificulta que os resíduos sejam etiquetados de forma padronizada, havendo ainda casos em que acabam sendo utilizadas etiquetas comuns.

Em vista destes resultados, foi proposta uma segunda etapa em nosso programa de gerenciamento de resíduos. Esta etapa prevê a informatização do sistema de solicitação das aulas práticas utilizando a metodologia de "workflow", que busca representar, através de um modelo computacional o processo de trabalho existente numa organização, monitorando eletronicamente o fluxo e a situação dos documentos envolvidos neste processo. Neste sistema, conectado à intranet do campus ou via internet, o professor irá solicitar as aulas práticas. Ao solicitar o material, terá a opção de procurar resíduos já cadastrados que possam ser utilizados como insumos. A solicitação somente será aceita se for totalmente preenchida, obrigando o professor a registrar os resíduos e propor o tratamento adequado ou reutilização dos mesmos. A Central de Materiais contará com um computador através do qual os funcionários poderão acessar as solicitações e imprimir automaticamente, e de forma padronizada, os rótulos para os resíduos indicados.

Esta etapa está em fase de implementação e um protótipo está em fase de testes, sendo que, após a conclusão do sistema, somente serão aceitas solicitações de aulas práticas por este meio. O sistema oferece ainda a vantagem de reduzir o preenchimento de formulários, pois permite o armazenamento histórico de aulas e experiências conduzidas. Acreditamos que, assim, conseguiremos um melhor gerenciamento dos resíduos, bem como maior participação dos professores no mesmo.

\section{CONCLUSÕES}

Muitas disciplinas da URI - Campus Erechim fizeram seu próprio gerenciamento de resíduos, contribuindo para o programa. O Gerenciamento de Resíduos proposto pela disciplina de Química Geral Experimental mostrou-se eficiente, proporcionando redução do consumo de reagentes e da produção de resíduos, aumento dos resíduos reaproveitados, diminuição dos rejeitos acondicionados e um descarte adequado para os rejeitos. Entretanto, houve alguns problemas que deverão ser eliminados com a informatização do sistema e espera-se que o Programa de Gerenciamentos de Resíduos da URI - Campus Erechim seja estendido a todos os laboratórios de ensino e de pesquisa.

\section{REFERÊNCIAS}

1. Amaral, S. T.; Machado, P. F. L.; Peralba, M. C. R.; Camara, M. R.; Santos, T.; Berleze, A. L.; Falcão, H. L.; Martinelli, M.; Gonçalves, R. S.; Oliveira, E. R.; Brasil, J. L.; Araujo, M. A.; Borges, A. C.; Quim. Nova 2001, 24, 419.

2. Cunha, C. J.; Quim. Nova 2001, 24, 424.

3. Alberguini, L. B.; Silva, L. C.; Rezende, M. O.; Quim. Nova 2003, 26, 291.

4. Izzo, R. M.; Chemical Heath \& Safety 2000, 29.

5. Abreu, D. G. de; Iamamoto, Y.; Quim. Nova 2003, 26, 582.

6. Afonso, J. C.; Noronha, L. A.; Felipe, R. P.; Freidinger, N.; Quim. Nova 2003, 26, 602 .

7. Bendassolli, J. A.; Máximo, E.; Tavares, G. A.; Ignoto, R. de F.; Quim. Nova 2003, 26, 612.

8. Cruz, T. Em Wokflow: A tecnologia que vai revolucionar processos; $2^{\mathrm{a}}$ ed., Atlas: São Paulo, 2000. 